\title{
INTENSITY INHOMOGENEITY CORRECTION SCHEME FOR 3D-DIMENSIONAL MRI BRAIN SCANS USING HISTOGRAM MATCHING
}

\author{
YAKUBU SULEIMAN BAGUDA ${ }^{1}$, ABUBAKAR SULEIMAN BAGUDA ${ }^{2}$ AND USMAN SULEIMAN BAGUDA ${ }^{3}$ \\ ${ }^{1}$ Department of Information Systems, King Abdulaziz University, Rabigh, Saudi Arabia. \\ ${ }^{2}$ Department of Psychiatric, Abubakar Tafawa Balewa University Teaching Hospital, Nigeria. \\ ${ }^{3}$ National Hospital, Abuja, Nigeria. \\ Email: bagudays@gmail.com
}

\begin{abstract}
Revised October 2016
ABSTRACT. There has been an explosive growth in medical imaging industry with rapid development in imaging techniques. Magnetic resonance imaging (MRI) has increasingly been popular as a result of its significant role in examining the ever-changing activity of human brain. The image and resolution produced by MRI is quite detailed and can detect tiny changes of structures within the brain and human body at large. It has become the leading technology for examining the living brain at work. More importantly, it plays an extremely important role in diagnosing patient with Alzheimer's disease in its early stage. In fact, this will dramatically assist in analyzing the brain scan images and identifying whether if there is any potential risk for developing Alzheimer's disease. Quantifying the brain for Alzheimer's patient on regular basis is very important so that to critically analyze and assess the rate at which the disease affect patient brain. However, the quality of the MRI scans can radically change due to environmental and atmospheric changes, and equipment ageing. Subsequently, this has a profound impact on the precision and accuracy of the measurement of the brain. In this paper, we proposes an approach which eliminates the in homogeneity problem anticipated so that $3 D$ brain MRI scans can be efficiently processed and analyzed.
\end{abstract}

Keywords: Intensity inhomogeneity; Magnetic resonance imaging; Alzheimer's; Brain Scan; Histogram matching.

1. Introduction. Magnetic resonance imaging (MRI) technology is an extremely important tool used to perform brain scans that show the structure of the brain, revealing shrinking or expanding of gray and white matter in the brain. More importantly, it clearly shows change in the brain's blood flow over time while individuals are performing particular tasks. Hence, MRI can play an integral role in medical imaging such that diseases like Alzheimer's can be diagnosed in its early. This will be extremely useful as means for curing more diseases such as Alzheimer's, schizophrenia etc. However, this work clearly demonstrates approach which eventually equalizes the MR image intensity so that the brain volume can be determined. Indeed, it is very obvious that human brain shrink as people get older, but the rate of shrinkage should not exceed certain limit. Alzheimer's has become a major disease which commonly affect the elderly people. The percentage of the people affected has dramatically increased in recent years and hence there is dramatic need for more intensive research and development to cure the disease.

MRI is primarily based on the principle of nuclear magnetic resonance. Resonance is nothing rather than an amplified stimulus that eventually has the same natural frequency. The nuclear magnetic resonance involves measurement of signals coming from nuclei in response to radio waves that have the same natural 
frequency as the nuclei themselves. In fact, hydrogen is the simplest and most abundant element in the human body. Every water molecule contains hydrogen atoms, and larger biological molecules such as lipids and proteins, contain many hydrogen atoms. A hydrogen atom consists of a proton nucleus and a single electron. Normally protons have a magnetic axis. In nature, the orientation of these axes is random. In the presence of a magnetic field, however, protons spin around an axis that is aligned with the main magnetic field. This spinning of protons within a magnetic field is referred to as precession. The dominant axis is oriented in magnetic field; slightly fewer spins are oriented in 180 degrees opposite direction. This equilibrium longitudinal magnetization can be considered potential energy. When the equilibrium is disturbed by an appropriate radio pulse, MR signals are generated. These signals can be measured and used to construct images. The frequency with which protons precess is directly proportional to the strength of the main magnetic field. This frequency is often referred to as resonance frequency because it is equal to the frequency of a radio pulse that induces resonance in the protons $[1,2,3]$.

Generally the image obtained from the aforementioned process could be of different intensity due to several reasons. When determining the brain volume, the image intensity has to be uniform. It is very challenging to ensure that two MR images taken at different time have the same intensity. Hence, this has led to more advance research in order to ultimately achieve uniformity in MRI brain images. There has been an indication that MRI in homogeneity correction started many years back [4,5]. Later, more extensive research work has been conducted on possible causes of intensity in homogeneity in MR images [6].

The remainder of this paper is organized as follows. Section 2 mainly focuses on the overview of magnetic resonance imaging. Section 3 introduces our proposed scheme. Simulations results are presented in Section 4. Finally, conclusions are enumerated in Section 5.

2. MRI Overview. The MRI instrument primarily includes one or more computers, a radio transmitter and receiver, one or more radio transceiver coils, magnetic field gradient coils, and the main magnet. The timing and strength of magnetic field gradients and radio pulses are controlled through an acquisition computer that uses parameters chosen by the MRI operator. The commands from the computer are sent to gradient and radio frequency amplifiers, which generate, respectively, gradient and radio pulses. The impulses for radio pulses are sent from the RF power amplifier to transmit and receive driver, which triggers the generation of the radio pulses. The resulting radio pulses are transmitted from an antenna, or coil, to excite the protons in the body part of interest [1].

In some cases, the same coil is used both to send and receive MR signals. In order instances, as for superficial structures, a separate coil, referred to as a receive-only coil, is used to receive the MR signals. The signals from the body part of interest are sent from the receiving coil back through the transmit and receive driver to a preamplifier, which prepares the signals for conversion to digital signal.

Initially, the receive signal consist $s$ of numerous analog radio waves that make up a composite waveform. This complex composite waveform contains many different frequencies, phases, and amplitudes. These data do not directly indicate the spatial location of the protons from which they originated. The MR signals undergo analog-to-digital conversion (ADC), producing digital data that consist of a set of numbers representing points along the waves. These data are filtered, stored in memory, and processed by the MR acquisition computer. The digital data are not mapped directly as point in a final image; rather, they are represented in a matrix referred to as k-space. Each point in k-space contains data from all portions of an MR image

2.1. Image Intensity. One of the major advantages of MRI compared to other imaging techniques is the excellent soft tissue discrimination of the images. The contrast characteristics of each image depend on many variables. An image has contrast if there are areas of high signal, as well as areas of low signal. Some areas have an immediate signal. A tissue has a high signal if it has a large transverse component of magnetization. If there is a large component of transverse magnetization the amplitude of the signal received by the coil is large resulting in a bright area on the image. A tissue returns a low signal, if it has a small transverse component of magnetization. If there is a small component of transverse magnetization the amplitude of the signal received by the coil is small resulting in a dark area on the image. This phenomenon requires an external effort to readily ensure equal distribution of pixels across the image.

2.2. Brain ImagesThe brain like all biological structures is in three dimensional. Any point on or inside the brain can be localized on three axes or planes - the $\mathrm{x}, \mathrm{y}$ and $\mathrm{z}$ axes or planes. The brain is often cut into pieces for further study. These slices are usually made in one of three planes: 
coronal plane, the axial plane or the sagittal plane [6]. The coronal plane and the horizontal plane are shown in this figure on the right. The coronal plane is also called the frontal plane. These terms are fairly straightforward and have the same meaning with respect to any part of the nervous system. Figure-1 shows the human brain for axial, sagittal and coronal images.

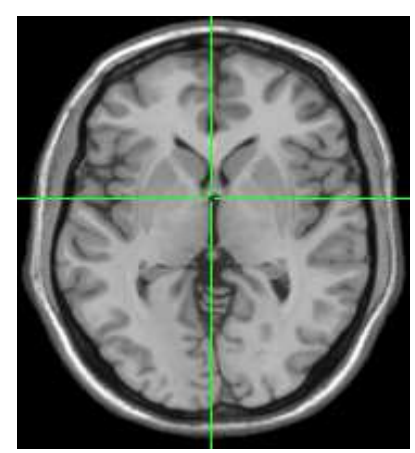

Axial Brain Image

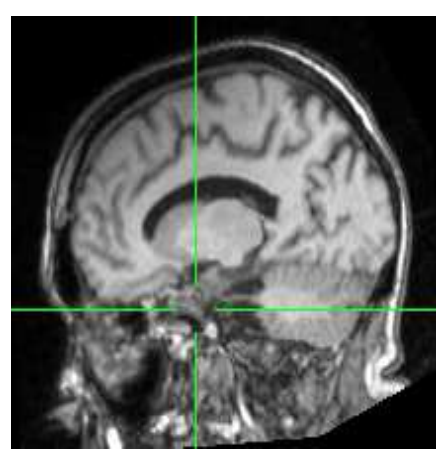

Sagittal Brain Image

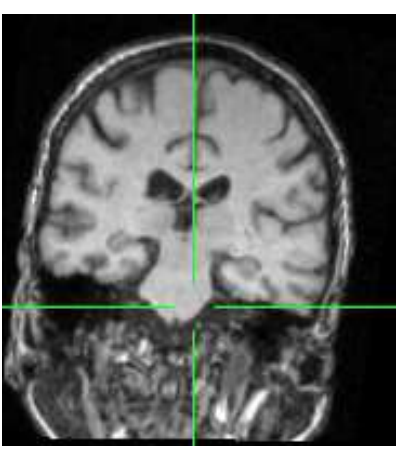

Coronal Brain Image

Figure-1: Human Brain in 3-planes

2.3. Three-dimensional Image Representation 3-D digital images can be represented as 3 dimensional array whose elements (voxels) represent samples of a certain physical quantity acquired over a usually rectangular 3-D grid. In general, 3-D simply refers to volume and volumetric change. The Figure below shows a typical 3-D image representation.

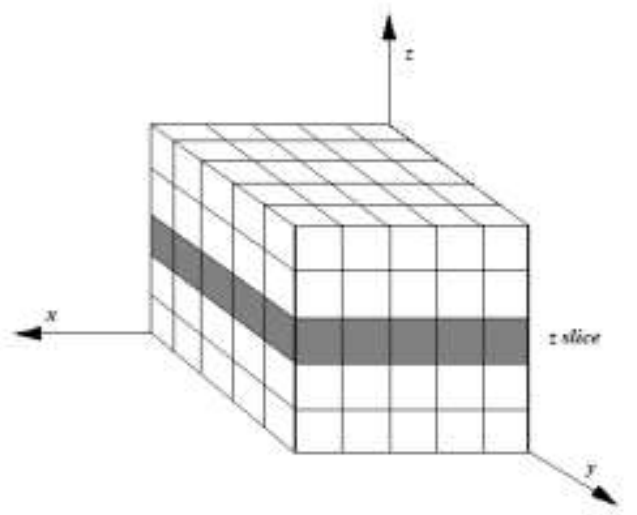

Figure-2: 3D Image Presentation

It has been mentioned already that a 3-D image is just like an ordinary 3-D matrix ( $(\mathrm{f}, \mathrm{x}, \mathrm{z}))$ of dimensions $\mathrm{D}_{1} \times \mathrm{D}_{2} \times \mathrm{D}_{3}$ where $\mathrm{x}, \mathrm{y}, \mathrm{z}$ represents the slice (image), row, and column coordinates respectively. This 3-D representation is very important since it is also applicable to the 3-D MRI scan of a human brain. Indeed, the distribution of voxels describes significant information about a 3-D image and its quality. The probability density function is extremely important in determining an image histogram. In this case, the histogram is determined by the probability density function of the voxels intensities. For instance, if to say the 3-D image has dimension D1 x D2 x D3 and having $\mathrm{k}$ discrete intensity level. The histogram at any intensity can be determine by using equation 1 below

$$
\mathrm{P}\left(\mathrm{i}_{\mathrm{k}}\right)=\frac{\mathrm{n}_{\mathrm{k}}}{\left(\mathrm{D}_{1} \times \mathrm{D}_{2} \times \mathrm{D}_{3}\right)} \quad \mathrm{k}=0,1,2 \ldots . \mathrm{L}-1
$$


Where $n_{k}$ is the number of voxels with intensity $\mathrm{i}_{k}$ and the number of levels $\quad \mathrm{L}=65536$ (16-bits ). In order to effectively handle the 3-D MRI brain images, the dimensions are adjusted to $182 \times 218$ $\mathrm{x} 100$. This subsequently allows clear visualization of the structure of the brain images. From the diagrams shown below, it can be seen that the two images are identical primarily because there were taken from the same patient and the same dimension.

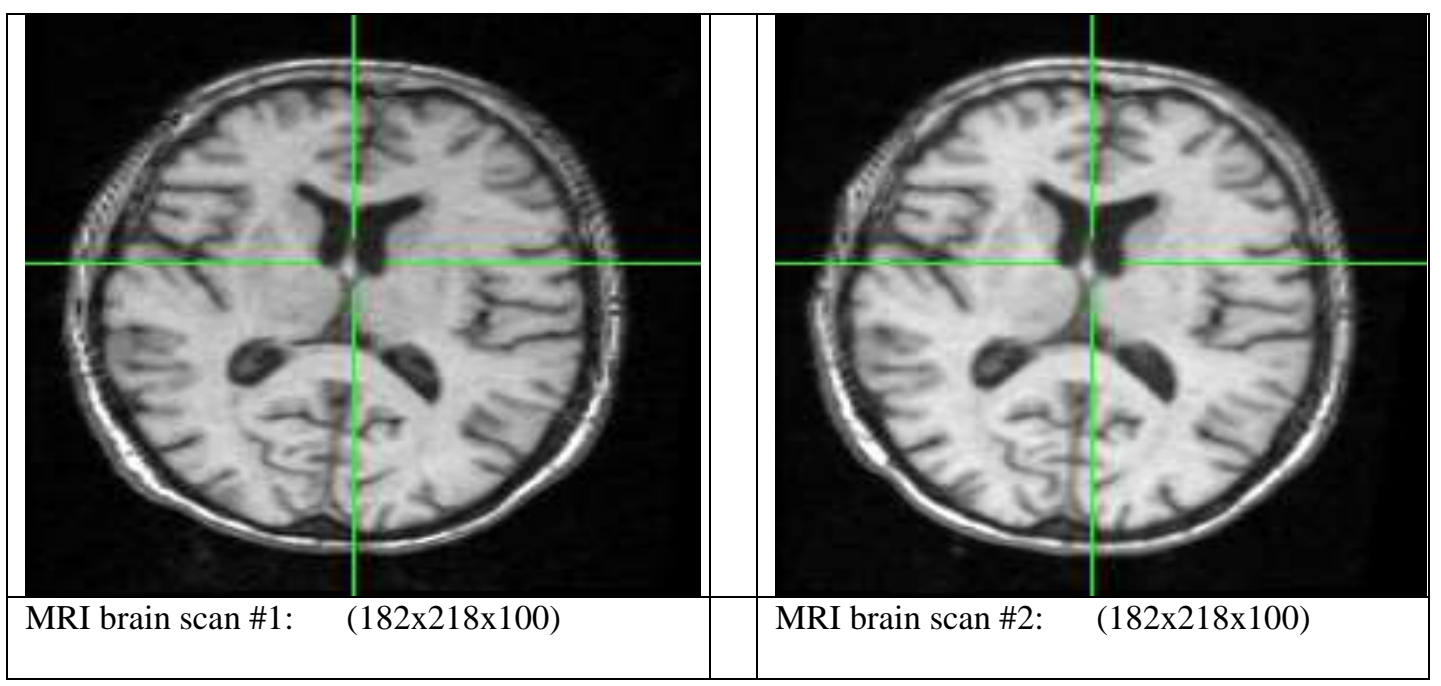

Figure-3: Sample Brain Images and their dimension

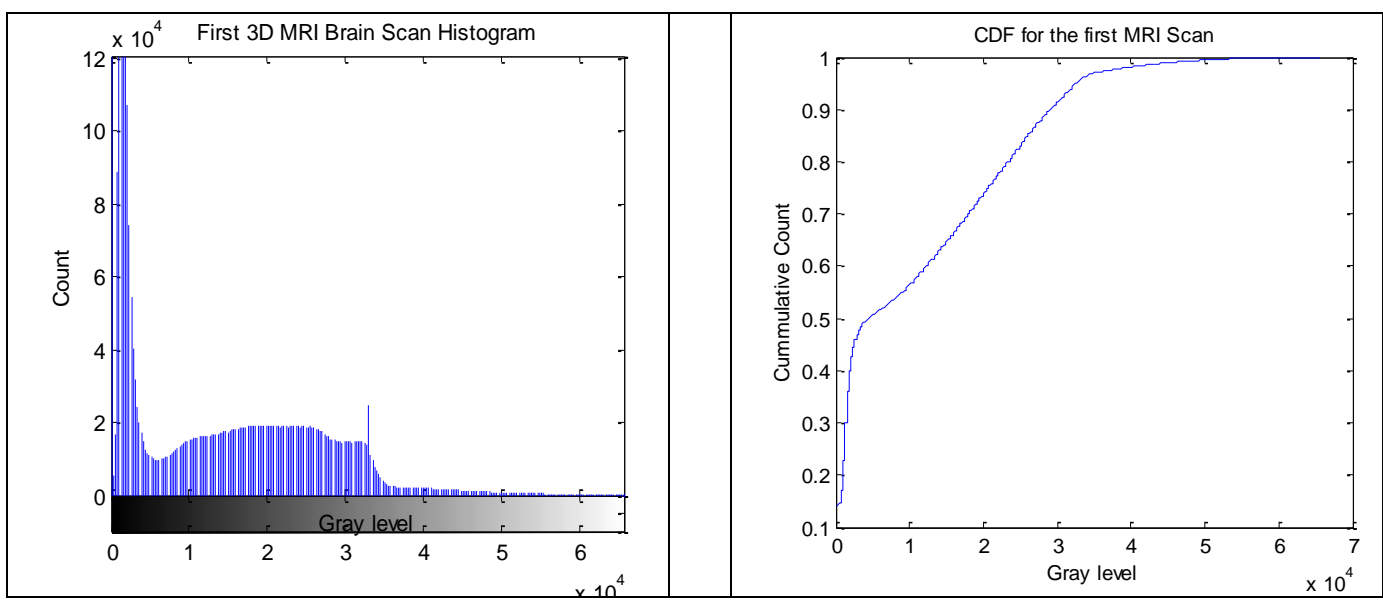

Figure-4: Histogram and CDF for the 3-D MRI Scan Image \#1 


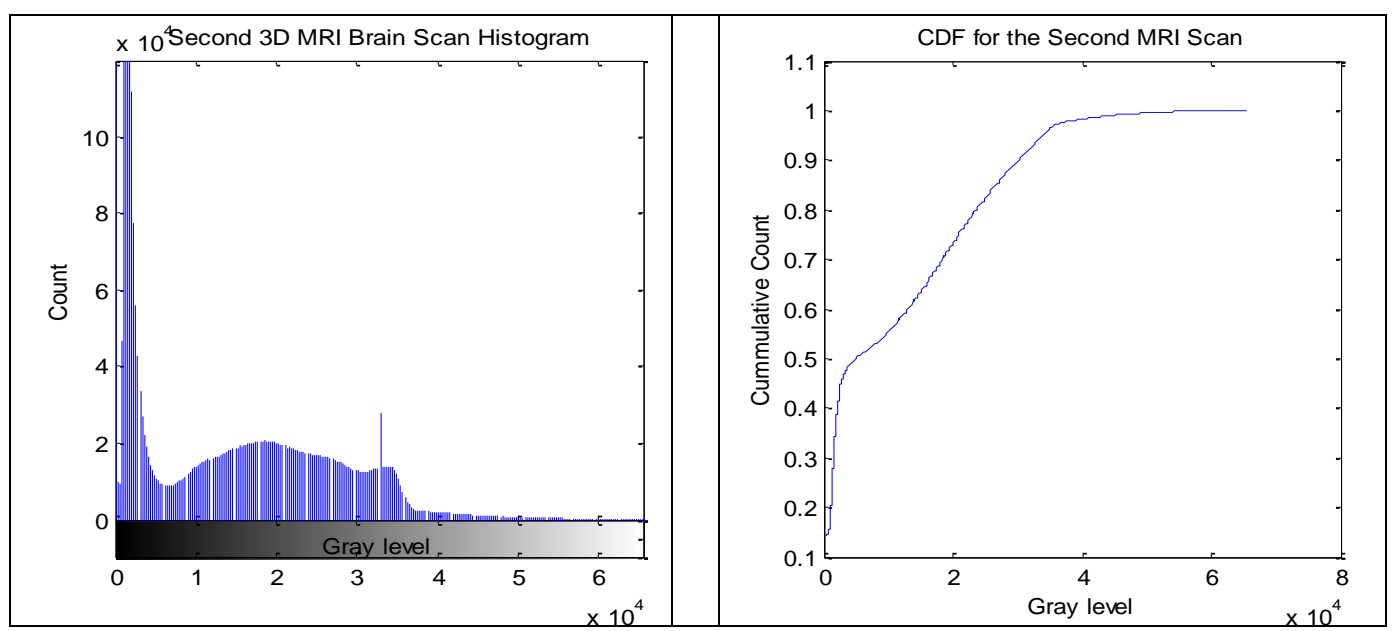

Figure-5: Histogram and CDF for the 3-D MRI Scan Image \#1

3. Methodology. Many techniques have been developed in order to significantly improve the intensity of MR images. Manipulating the MR image histogram has been one of the common approach used to mitigate the intensity in homogeneity problem. The histogram approach can be categorically divided into 3 main approaches namely [4]: Histogram Matching or Equalization, information minimization and high frequency maximization. The histogram equalization $[6,7]$ normally divides the MR image into sub-volume and each sub-volume intensity is represented in form of histogram. Basically, the intensity is maintained reasonably constant. Our experimentation primarily uses this approach to match the intensity of different MRI brain taken at different time from the same patient. Later, more detailed on how it has been used to achieved histogram matching will be discuss. This approach is purely based on the fact that the intensity in homogeneity corruption can eventually result in additional information to the in homogeneity free image. Therefore, the in homogeneity effect can be mitigated by constrained minimization of image information. In [8,9], MRI intensity in homogeneity correction has been proposed. The entropy and field smoothness constraints were minimized by using fast annealing. This approach primarily uses iteration and it normally smoothes the field which eventually has the high frequency content of the distribution. Perhaps the technique can be applicable to any MR image and proved to be highly efficient and effective. More importantly, the method is highly reliable such that no any improvement has been suggested due its sophistication [5].

A simple way of redistributing the voxels is based on the normalized cumulative histogram which is defined by the expression below. The normalized cumulative histogram can be used as a mapping between the original gray levels in the image and the new gray levels required for enhancement [7]. The cumulative density function can be determine using the expression given below

$\mathrm{P}\left(\mathrm{i}_{\mathrm{k}}\right)=\frac{1}{\left(\mathrm{D}_{1} \times \mathrm{D}_{2} \times \mathrm{D}_{3}\right)} \sum n_{i}$

It is primarily based on this function all the necessary manipulations can be made in order to achieve desired goal. Using the above formula, the cumulative density function of each the sample 3-D MRI brain scans can be determine for each gray level (65536). When these values are plotted the gray level subsequently yields the graphs shown in the figure below.

The above MRI scans doesn't actually has the same intensity due to the fact that each of the 3-D MRI scans was taken differently. This has contributed toward making the two 3-D brain images to have different intensity distribution. It has been known that the primary object is to ensure MRI brain scan taken from a patient at different time has the same intensity distribution. This can be achieved using histogram matching so that they both have the same histogram. In the next step, all the above methods so far discussed on histogram manipulation will be used to implement the algorithm which conveniently ensures proper histogram matching. The information contained in the histogram can be used to enhance the image quality. 
Initially, let us assume that $\mathrm{a}$ and $\mathrm{b}$ are the two continuous gray levels of the two images to be match and let $\mathrm{P}_{\mathrm{a}}(\mathrm{a})$ and $\mathrm{P}_{\mathrm{b}}(\mathrm{b})$ denote the probability density function of the 3-D MRI brain images. Lets a and $\mathrm{b}$ denotes the gray levels of the input and output images. $\mathrm{P}_{\mathrm{a}}(\mathrm{a})$ can be estimated from the original image and $\mathrm{P}_{\mathrm{b}}(\mathrm{b})$ is the specified probability density function which the output image is expected to have. The histogram equalization enhanced the contrast by transforming the voxels intensities such that the histogram of the output image is uniform.

For instance, a 3D image can be transform using the procedure below. Actually the point wise intensity transformation function $\mathrm{g}(\mathrm{x}, \mathrm{y}, \mathrm{z})=\mathrm{T}(\mathrm{f}(\mathrm{x}, \mathrm{y}, \mathrm{z}))$ that achieves uniform histogram for $\mathrm{g}(\mathrm{x}, \mathrm{y}, \mathrm{z})$ is given by equation 3 below

$$
\mathrm{g}=\mathrm{T}(\mathrm{f})=\int \mathrm{P}_{\mathrm{r}}(w) d w
$$

if $P_{r}$ is the histogram of $f$ and image intensities are set to a range between 0 to 1 . This can be applicable to each of the two images to be match. In this case, the 3D original image has a histogram function $P a$ is to be transformed into target 3D image with histogram function $P b$. The mapping for these functions can be achieved using the expression below.

$$
\mathrm{b}(\mathrm{x}, \mathrm{y}, \mathrm{z})=\mathrm{G}^{-1}[T(a)]
$$

The figure below shows diagrammatically how the process of histogram matching can be achieved. In a nutshell, it demonstrates all the steps involves in implementing the algorithm.

In summary, the 3-D MRI brain images histogram matching can be implemented using the algorithm below:

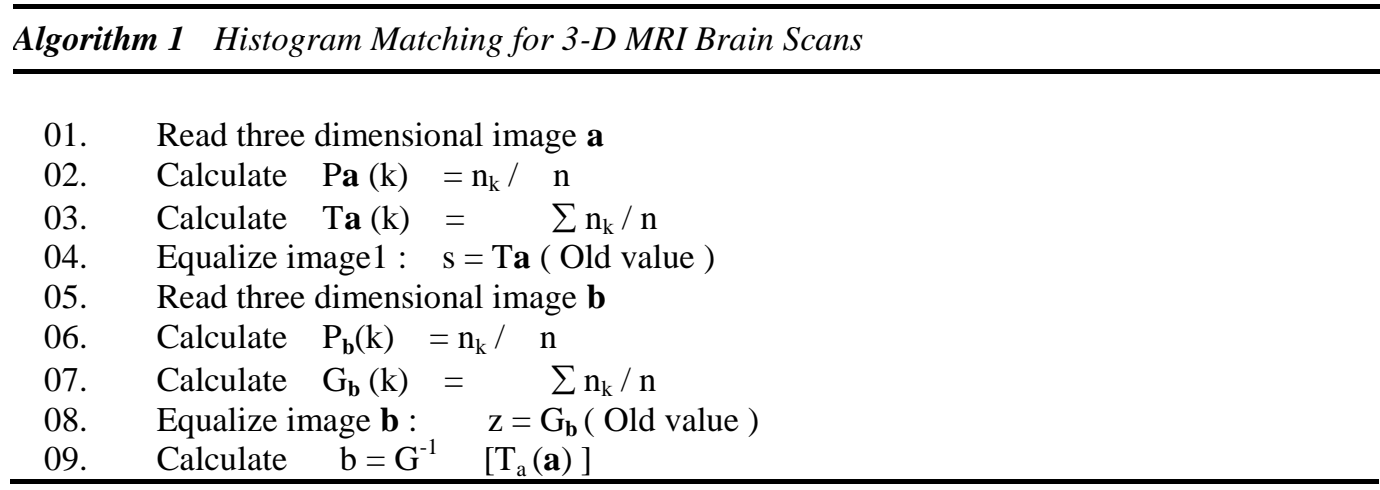

4. Numerical results. . In this research work, the dimension of the brain has been considered to be $182 \times 218$ $x$ 182. The figure below shows a typical 3-D MRI brain scan with the above dimensions. Different slices of the brain image can be obtained by adjusting the dimensions. 


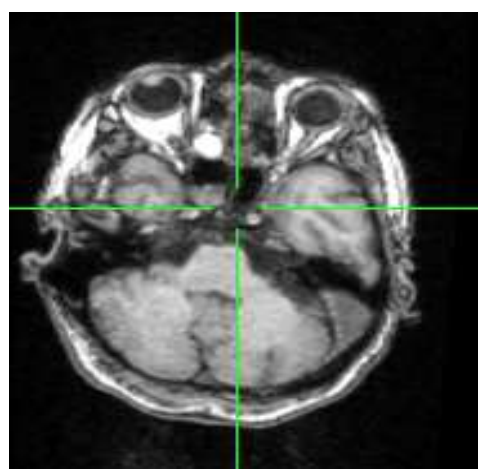

First 3-D MRI Brain scan

Dimension: $182 \times 218 \times 182$

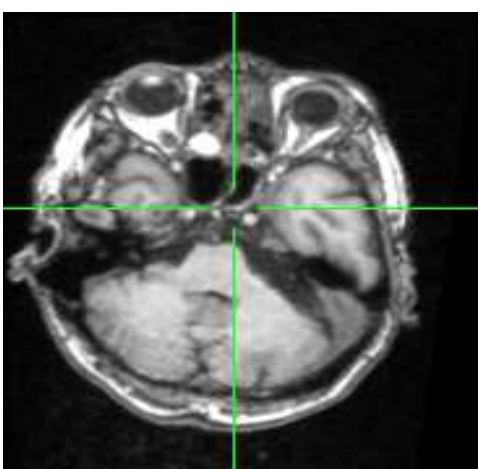

Second 3-D MRI Brain scan

Dimension: 182 x 218 x 182

Figure-6: Sample 3-D MRI Brain images

The two samples of the 3-dimensional images of the brain were acquired using a magnetic resonance imaging (MRI) scanner. 3-D MRI has been considered to be more useful because of the fact that it represents the human in terms of size and shape but it is more complex to manipulate when compared to ordinary 2-D images. MRI is a flexible medical imaging tool which allows imaging of an arbitrary volume. The MRI data sets are images of proton density, and are displayed as 16-bit grayscale images. It is common to acquire images of the brain in the form of many consecutive 2-dimensional slice images. Each slice covered an area $218 \times 182$, with 65536 pixels in the axial and coronal directions. Thus the entire 3-dimensional image was 182 (Saggital) x 218 (Axial) x 182 (Coronal). Initially, only 218 x 182 of the brain image was considered since it is assumed that after equalizing the 2-D MRI brain scans, the 3-D MRI could be handle more properly using the same method used for 2-D since it only require little modifications.

The method used for testing the capability and effectiveness of the algorithm developed which eventually equalized the histogram of two different MRI scans is extremely important. Both the two samples of the MRI scans were tested using the algorithm developed in order to demonstrate the effectiveness of the histogram matching scheme. The approach used for the 2-D MRI slices and complete brain images was the same but little modifications were made in the program so that it could conveniently equalized the histogram for 3-D MRI images. Firstly, the 2-D slice would be considered as part of the effort to have clear view of the main 3-D MRI scans. While conducting the test on the slices, one of the slices was matched to the other and the results were noted. Again, one of the slices was matched to a reference distribution which is determined by the function used as the reference. The dimension used for both the images was symmetrical so that any negligible change can be detected. Figure 5-2 shows how histogram matching of 3D MRI is achieve using histogram equalization or modification. In order to enhance the performance of the program, it is segmented into part such that any problem anticipated can be traced.
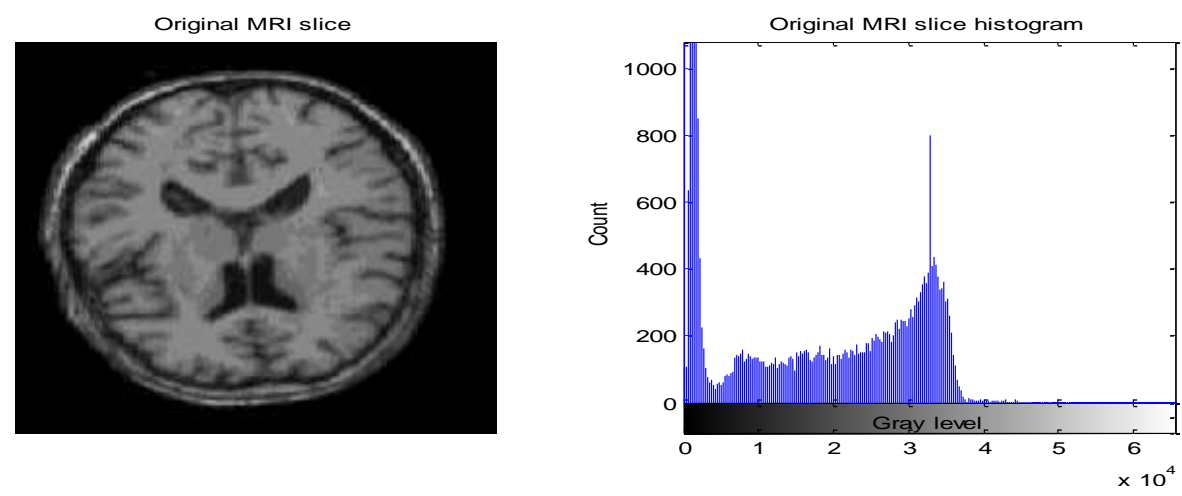

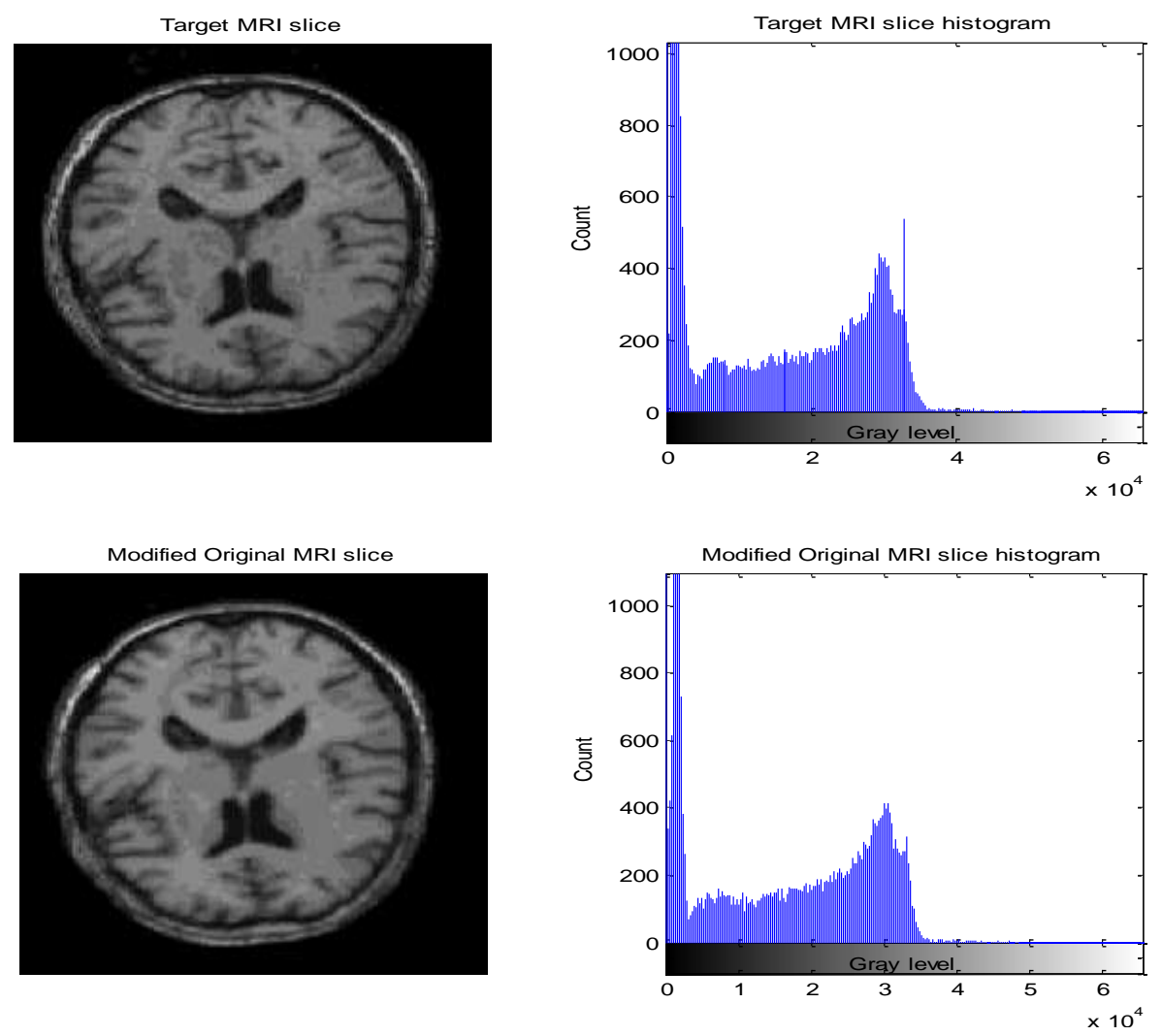

Figure-7: Brain Slice histogram matching

As it can be seen in figure 7, both the brain slice and 3D MRI brain were matched in order to obtain the desired target image, but it is difficult to achieve perfect matching due to lost of some pixels during histogram matching. The issue of perfect matching is extremely important in order to obtain optimum result. More precise and accurate matching can be achieved by ensuring that each of the pixels or voxels lost in the process of matching of the two images have been recovered. Obviously, this can be implemented by replacing any of the pixels with its neighboring pixel in order to fill the gap in between the values. This involves the use of crude approximations and manipulation.

Conclusion. In this paper, an approach for correcting intensity nonuniformity in 3D MRI brain scan has been described. This correction approach will assist in analyzing and evaluating the patient's brain so that the probability of person to be affected by cronic brain diseases could be determined by the shrinking rate. This method of histogram analysis has a variety of applications such as image registration, brain segmentation and tissue classification. The developed approach reduces the detrimental impact of the spatial resolution on the MRI brain scan images and it consequently allows effective interpretation and quantitative analysis of the images. More importantly, the structures of the MRI scans can be studied and analyzed more efficiently.

\section{REFERENCES}

[1] Bankman, I. N., \& Morcovescu, S. (2002). Handbook of Medical Imaging. Processing and Analysis. Medical Physics, 29(1), 107-107.

[2] Gillespie, J. E., \& Jackson, A. (Eds.). (2000). MRI and CT of the Brain. Arnold.

[3] Westbrook, C. and Kaut, C, (1998). MRI In Practice, $2^{\text {nd }}$ ed., Blackwell Science.

[4] Yang, J., Huang, S. C., Mega, M., Lin, K. P., Toga, A. W., Small, G. W., \& Phelps, M. E. (1996). 
Investigation of partial volume correction methods for brain FDG PET studies. IEEE Transactions on Nuclear Science, 43(6), 3322-3327.

[5] Vovk, U., Pernus, F., \& Likar, B. (2007). A review of methods for correction of intensity inhomogeneity in MRI. IEEE transactions on medical imaging, 26(3), 405-421.

[6] Shattuck, D. W., Sandor-Leahy, S. R., Schaper, K. A., Rottenberg, D. A., \& Leahy, R. M. (2001). Magnetic resonance image tissue classification using a partial volume model. Neurolmage, 13(5), 856-876.

[7] Gonzalez, R. C., \& Woods, R. E. (2002). Digital Image Processing, A. Dowrkin, Ed. Upper Saddle River, New Jersey 07458.

[8] Likar, B., Viergever, M. A., \& Pernus, F. (2001). Retrospective correction of MR intensity inhomogeneity by information minimization. IEEE transactions on medical imaging, 20(12), 1398-1410.

[9] Mangin, J. F. (2000). Entropy minimization for automatic correction of intensity nonuniformity. In Mathematical Methods in Biomedical Image Analysis, 2000. Proceedings. IEEE Workshop on (pp. 162-169). IEEE. 